\title{
Algebraic Approach to the Description of Temporal Knowledge in Decision Support Tasks
}

Levykin Viktor $^{1}$

Chala Oksana $^{1}$
${ }^{1}$ Kharkiv National University of Radio Electronics, 14 Nauky Ave, KharkivUA-61166, Ukraine, viktor.levykin@nure.ua

\begin{abstract}
The problem of automated construction of a temporal knowledge base for supporting decision-making in solving partially structured and unstructured organizational management problems is considered. The solution to this problem is associated with the formalization of temporal relations, reflecting the implemented sequence of control actions. The formal description should take into account not only the relationship in time between control actions, but also the static dependencies between the structural elements of the organizational management object, represented by the organizational structure of the object. An algebraic approach to the description of temporal knowledge is proposed. According to this approach, common temporal rule is defined. The rule determine the relationship in time between facts. Each logical fact is knowledge about the state of the control object at a certain point in time. The rule establishes temporal dependencies for time-consistent facts, as well as for facts, which are true at certain intervals. The proposed algebraic approach makes it possible to combine temporal relations that reflect the control process with dependencies, taking into account the structure of the control object.
\end{abstract}

Keywords: management decision, decision support, temporal relationship, state of the control object, algebraic approaches.

\section{INTRODUCTION AND PROBLEM STATEMENT}

Decision support is used to solve partially structured and unstructured tasks at the tactical and strategic levels of organizational management. Such support is carried out in order to form a management decision for atypical states of the control object. The specified solution is implemented in conditions of incomplete information about the current state and includes a sequence of control actions that ensure the transition from the current to the target state of the control object.

The sequence of management decision involves solving the problems of finding the set of possible sequences of control actions, as well as implementing the selected decision. The first task involves the subtasks of detecting and analyzing the anomalous state of a management object, as well as generating many alternative options for executing a management decision. Solving the sub-problem of constructing multiple alternatives requires considerable resources. This determines the importance of using the decision support system in solving this problem. The second problem is solved directly by a decisionmaker and combines the choice of one of the alternatives and the implementation of the selected decision.

The search for managerial decisions is usually implemented in decision support systems [1] using a knowledge base. Existing approaches to construction a knowledge base in such systems are based on the use of communicative methods for extracting knowledge. These methods provide for the formalization of the knowledge necessary to support decisions based on the results of a dialogue with experts in a selected subject area [2]. However, communicative methods are timeconsuming. It does not allow timely to obtain an up-to-date knowledge base in the event of an evolutionary change in approaches, methodologies and technologies that are decisive in the activities of the enterprise. Such a development of technology is characteristic of modern innovative enterprises, for example, in the field of software development for information systems.

To overcome the key disadvantage of traditional approaches to construction a knowledge base, it is advisable to use temporal relations instead of causal ones [3]. The latter determine the temporal ordering of control actions. Therefore, they can be obtained on the basis of the analysis of the sequence of actions as part of the implemented management decisions. Such an opportunity creates the conditions for the automated construction of temporal knowledge bases in decision support systems $[4,5]$.

The relevance of the research topic is related to the fact that solving the problem of automated formation of a knowledge base based on the analysis of temporal ordering of known sequences of control actions requires the construction of a formal basis for describing temporal knowledge. The specified description should reflect both the temporal relationship between the control actions and the static dependencies represented by the organizational structure of the control object.

The purpose of the report is to construction a formal description of temporal knowledge in order to provide the possibility of the formation and verification of many alternative options for managerial decisions in the form of time-ordered sequences of control actions.

\section{INTRODUCTION AND PROBLEM STATEMENT}

Algebraic approaches to the description of algorithms and information technologies have found application in improving the structure of software systems in order to increase their speed, as well as identifying deadlock states.

An algebraic description of knowledge, including the rules of inference, makes it possible to formalize the processes of building and expanding the knowledge base in decision support tasks. Such a description contains algebraic structures represented by a set with relations defined on it. The first, a static relation, defines the basic, unchanging relationships between the elements of the set. The second relationship determines the dynamics of change in the implementation of organizational management.

The developed algebraic description of temporal knowledge reflects the many states of the control object, as well as the hierarchical and temporal relations between these states. 
The state of a control object at each time is determined by a finite set of attributes $A$. The typed values of each element $a_{k} \in A$ are given by a finite set $V_{k}=\left\{v_{k, l}\right\}$. The acquisition of a variable $a_{k}$ the value $v_{k, l}$ within the proposed description of temporal dependencies is displayed as an elementary fact $\varphi_{k, l}$.

Every fact $\Phi_{j}$, that reflects the state $s_{j}$ of a control object at a point in time $\tau_{j}$, is given in the representation of temporal knowledge through conjunction of elementary facts $\varphi_{k, l}, k=\overline{1, K}$. If all the facts $\varphi_{k, l}$ are true at a point in time $\tau_{j}$, then the fact $\Phi_{j}$ is also true:

$$
\Phi_{j}=\left\{\begin{array}{l}
\text { true if } \exists \tau_{j}: \forall k \varphi_{k, l}=\text { true } \\
\text { false otherwise. }
\end{array}\right.
$$

The set $\Phi=\left\{\Phi_{j}\right\}$ is partially ordered by the moment $\tau_{j}$ of truth of these facts. Facts reflecting one alternative sequence of control actions $\Pi_{i}$ are linearly ordered in time. Facts belonging to different sequences $\Pi_{i}$ may not have temporal relationships.

Each pair of facts $\left(\Phi_{j}, \Phi_{m}\right)$, pertaining to one alternative is always associated with a temporal relation. Such a pair of ordered facts in the knowledge base seems to be a temporal rule.

The first fact is the antecedent of the rule and determines the state of the control object, which is a condition for the implementation of the temporal rule. The second fact is a consequence of the rule. Consequence defines a control action and its results as a new state of the control object. The state $s_{m}$, is reflected by the fact $\Phi_{m}$. Accordingly, the application of the temporal rule changes the set of facts that are true at the current time. The relationship between the facts is set by the temporal operator $o \in O$ and temporal quantifier.

Temporal operators $O$ determine the set of possible temporal relationships between facts. For example, a fact $\Phi_{m}$ must definitely become true immediately after the fact has become true $\Phi_{j}$. Or else the fact $\Phi_{m}$ will be true sometime in the future after the fact $\Phi_{j}$.

The sequence of revealing the true facts, applying the temporal rules, as well as forming a new list of the true facts is the basis of a direct output on the temporal rules. The result is a sequence of states of a management object, or a sequence of control actions within a management decision.

Static structural dependencies between facts are determined by the set $H$ of operators of union, intersection, and fact difference. For graphical representation of these operators are used traditional signs of combination, intersection of sets: $\{\bigcup, \cap, \backslash\}$.

The union of facts $\Phi_{j}, \Phi_{m}$ is performed when they determine the status of the individual components of the control object. The condition of a control object as a whole is generally determined as a union of these facts:

$$
\Phi_{j}^{(1)} \cup \Phi_{j}^{(2)}=\left(\bigwedge_{k: a_{k} \in A_{1} \cup A_{2}} \varphi_{k, l}\right) \mid \tau \geq \tau_{j}^{(1)}, \tau \geq \tau_{j}^{(2)},
$$

where $\tau$ - current time, $A_{1}, A_{2}$ - sets of attributes that determine the state of the control object represented by facts $\Phi_{j}^{(1)}$ and $\Phi_{j}^{(2)}$, respectively.

According to (2), the united fact will be true when the last of the facts $\Phi_{j}^{(1)}, \Phi_{j}^{(2)}$ is true.

The intersection and difference of facts is defined similarly.

In general, the union and intersection of logical facts makes it possible to use «bottom-up» and «top-down» approaches to construct a hierarchical representation of temporal knowledge.

Algebraic description $\mathcal{R}$ of temporal knowledge for decision support tasks consists of a basic set of facts, as well as relationships that determine the static and dynamic relation between these facts:

$$
\mathcal{R}=\left\{\Phi, O, H: \forall\left(\Phi_{j}, \Phi_{m}\right) \in \Phi \exists(o \vee h)\right\},
$$

where $\Phi-$ set of facts $\Phi_{j}$, that describe state of the control object; $o \in O$ - temporal operator that determines the type of temporal relationship between the facts; $h \in H$ - set of relationships that allow you to determine the hierarchy of these facts, including the organizational structure of the control object.

\section{CONCLUSIONS}

A generalized description of temporal knowledge is proposed. The description defines the time dependencies between the facts presented in the form of temporal rules. Each fact corresponds to the knowledge of the condition of the control object at a certain point in time. The state of a control object is defined by set of values of variables that describe that object. Each rule establishes temporal relations for a pair of facts. These facts can be true both consistently over time and at intervals.

In practical terms, the proposed algebraic approach makes it possible to take into account the following features of the use of knowledge to support decision-making: the application of personal knowledge of performers in addition to the formal knowledge describing the behavior of the control object; multilevel organization of knowledge, reflecting the organizational hierarchy of the control object; expansion knowledge as the management process is implemented.

\section{REFERENCES}

[1] C.K. Oduoza. "Decision support system based on effective knowledge management framework to process customer order enquiry", in Chiang, S. Jao(Eds), Decision Support Systems, INTECH, Croatia, 2010, p. 406.

[2] K. Dalkir "Knowledge Management in Theory and Practice", Burlington, Massachusetts: Elsevier Butterworth-Heinemann, 2005, 372 p.

[3] V. Levykin, O. Chala "Method of determining weights of temporal rules in markov logic network for building knowledge base in information control system", EUREKA: Physics and Engineering, 2018, №.5, pp. 310. DOI: http://dx.doi.org/10.21303/2461-4262.2018.00713.

[4] V. Levykin, O. Chala (2018). Method of automated construction and expansion of the knowledge base of the business process management system. EUREKA: Physics and Engineering, 4, 29-35. doi: 10.21303/2461-4262.2018.00676.

[5] Levykin, V., Chala, O. (2018). Development of a method for the probabilistic inference of sequences of a business process activities to support the business process management. Eastern-European Journal of Enterprise Technologies, 5 (3 (95)), 16-24. doi: https://doi.org/10.15587/1729-4061.2018.142664 